\title{
Guidelines for the Conservation of Subnival Wet Grassland Plant Formation of the Moor, 'Rio Blanco' in the Chimborazo Fauna Produc- tion Reserve
}

VII International Congress of

Science, Technology,

Entrepreneurship and

Innovation (SECTEI 2020)

Corresponding Author:

G. M. Ati

guicela.ati@espoch.edu.ec

Published: 26 August 2021

Production and Hosting by

Knowledge E

(c) G. M. Ati. This article is distributed under the terms of the Creative Commons

Attribution License, which permits unrestricted use and redistribution provided that the original author and source are credited.
S OPEN ACCESS

\section{Lineamientos para la Conservación de la For- mación Vegetal Herbazal Húmedo Subnival del Páramo, 'Rio Blanco' en la Reserva de Producción de Fauna Chimborazo}

\author{
G. M. Ati, E. A. Muñoz, D. A. Vistin, and G. Y. Balseca
}

Facultad de Recursos Naturales, ESPOCH, Riobamba, Ecuador

\section{Abstract}

The Chimborazo Wildlife Production Reserve is one of the most important protected areas in Ecuador, located in the provinces of Chimborazo, Tungurahua, Bolívar in the Andes, has an area of 52683 ha, consisting of eight plant formations, 41 indigenous communities that inhabit in this conservation site. Rio Blanco, is a representative community of the subnival wet grassland ecosystem of the moorland where the research was carried out, in which the floristic composition, the threats were analyzed, from which the guidelines for its conservation were formulated. The Gloria methodology was applied to determine coverage, biodiversity indexes and the IVI. 10 families and 23 genera were found, the most abundant are asteraceae and geraniaceae. The site reports a diversity that goes from medium to high. The identified ecosystem services are supply, regulation and cultural. From the analysis of contribution and irreversibility; of severity and scope, the formation presents an average of (2.05) and (2.8) respectively, it means that the pressure is wide in scope, with these elements in a participatory way the guidelines were defined to avoid the deterioration of the ecosystem to Through four programs.

Keywords: conservation, training, grass/and threats, guidelines.

\section{Resumen}

La Reserva de Producción de Fauna Chimborazo es una de las áreas protegidas más importantes de Ecuador, ubicada en las provincias de Chimborazo, Tungurahua, Bolívar en los Andes, tiene una extensión de 52683 ha, formada por ocho formaciones vegetales, 41 comunidades indígenas que habitan en este sitio de conservación. Rio Blanco, es una comunidad representativa del ecosistema herbazal húmedo subnival del páramo donde se desarrolló la investigación, en esta se analizó la composición florística, las amenazas, a partir de lo cual se formularon los lineamientos para su conservación. Se aplicó la metodología Gloria para determinar coberturas, índices de biodiversidad y el IVI. Se encontraron 10 familias y 23 géneros, las de mayor abundancia son asteraceae y geraniaceae. El sitio reporta una diversidad que va de media a alta. Los servicios ecosistemicos identificados son de suministro, regulación y cultural. A partir de los análisis de contribución e irreversibilidad; de severidad y alcance, la formación presenta un promedio de $(2,05)$ y $(2,8)$ respectivamente, significa que la presión es 
de amplio alcance, con estos elementos en forma participativa se definieron los lineamientos para evitar el deterioro del ecosistema a través de cuatro programas.

Palabras Clave: conservación;, formación, herbazal, amenazas, lineamientos.

\section{Introducción}

En una época donde la pérdida de biodiversidad se ha acelerado uno de los mayores logros de la humanidad es la creación de áreas protegidas -AP- [1], que sirven como estrategia para la conservación de la biodiversidad y para la mitigación de los efectos negativos de la interacción sociedad-naturaleza [2, 3] son herramientas trascendentales para la gestión y protección de los ecosistemas [4], han permitido establecer las mayores superficies globales dedicadas a la conservación [2]; las zonas con mayor dinamismo en la creación de nuevas AP son Centro América y el Caribe.

A pesar de los esfuerzos generalizados para la conservación un tercio de la tierra protegida mundial está bajo una intensa presión humana [1], se prevé que estas presiones sobre las AP y el conflicto entre la conservación de la biodiversidad y las necesidades de la población local aumenten debido a numerosos factores [5] como la degradación ambiental, la reducción de tamaño de las AP, y una dinámica socioeconómica difícil de controlar [6, 7].

Dilucidar la importancia de las comunidades locales en las acciones de protección es fundamental para la conservación a largo plazo [8], por lo tanto es necesario gestionar las áreas protegidas incluyendo los aspectos del bienestar humano, incorporando a la población local en su gestión a través de un enfoque ecosistémico que promueva además su uso sustentable de forma justa y equitativa [9], en este contexto la conservación se considera un acontecimiento social [10].

Las áreas alto andinas presentan elevada vulnerabilidad al desequilibrio ecológico provocado por factores antrópicos y naturales, bajo esta perspectiva es imprescindible contar con información de la tipología de la vegetación, con el propósito de generar estrategias para la mitigación y adaptación a los efectos provocados por los factores anteriormente citados, de tal forma que los impactos sobre la población sean minimizados e, incluso, puedan ser aprovechados para mejorar las condiciones de vida [4, 11-13].

Ecuador cuenta con 56 áreas dedicadas a la conservación y protección de ecosistemas en los niveles terrestre y marino, a la provisión de servicios eco sistémicos 
[14], una de las área protegidas más importantes del país es la Reserva de Producción de Fauna Chimborazo con una extensión de 52683 [15], ubicado en los andes ecuatorianos en la que habitan 41 comunidades indígenas $[16,17]$ compuesta por ocho formaciones vegetales: Herbazal del Páramo, Herbazal y Arbustal siempreverde subnival del Páramo, Arbustal siempreverde y Herbazal del Páramo, Herbazal inundable del Páramo, Herbazal ultrahúmedo subnival del Páramo, Bosque siempreverde del Páramo, Herbazal húmedo subnival del Páramo, Herbazal húmedo montano alto superior del Páramo [4, 11, 17].

La formación vegetal herbazal húmedo subnival del páramo se caracteriza por la presencia de pastos de tallo corto, rosetas acaulescentes y hierbas en cojín, una comunidad característica de esta formación vegetal son los cojines edafoxerófilos [19], que son un grupo diverso adaptado fisiológicamente a las condiciones climáticas extremas [20-22]. Esta formación vegetal ofrece una serie de servicios eco sistémicos [23], no obstante en la actualidad son reconocidas como fuentes críticas de agua con grandes reservas de carbono en el suelo y altos niveles de biodiversidad [24], por ello se consideran ecosistemas estratégicos para la población rural y para las poblaciones urbanas [24]. Dada la importancia de este tipo de vegetaciones es esencial su conservación para la gestión de áreas protegidas.

En la Reserva de Producción de Fauna Chimborazo las 41 comunidades en su mayoría indígenas han tenido constante interacción [23] con esta formación vegetal a lo largo de los años, la comunidad Rio Blanco está circunscrita a la parroquia Pilahuin [25], ocupa gran parte de la formación vegetal herbazal húmedo subnival del páramo.

Las principales actividades de subsistencia de la comunidad son la agricultura y ganadería $[11,17,26]$ lo que ha generado que con transcurrir de los años la expansión de áreas cultivables y las practicas pecuarias como la quema y pastoreo se contraponen a los objetivos de conservación del área natural protegida [17, 26, 27].

El proyecto 'Medidas ante los riesgos que afrontan los ecosistemas de la Reserva de Producción de Fauna Chimborazo frente al cambio de uso de suelo que ejecuta la Escuela Superior Politécnica de Chimborazo' como parte de su acción investigativa viene trabajando en la formación vegetal herbazal húmedo, uno de los territorios de estudio es la comunidad Rio Blanco en la cual se determinó la composición y diversidad florística mediante la aplicación del método GLORIA [28], se identificaron las amenazas significativas que afectan a la formación mediante el método desarrollado por The Nature Conservancy [29], a partir de estos resultados se construyó en forma participativa los lineamientos para su conservación. 


\section{Materiales y Métodos}

\section{1. Área de estudio}

La investigación se realizó en la Reserva de Producción de Fauna Chimborazo en la formación vegetal herbazal húmedo subnival del páramo [17, 30] en la cual se identificaron 10 parcelas de muestreo (Tabla 1 y Figura 1). Esta zona está localizada en la provincia de Tungurahua, cantón Ambato, parroquia Pilahuin, Comunidad Rio Blanco que se encuentra localizada en las coordenadas 746272 E y 98475050 S (en metros) y a una altitud de 4350 metros sobre el nivel del mar (Tabla 1).

\section{Table 1}

Ubicación de las zonas de muestreo dentro de la RPFCH.
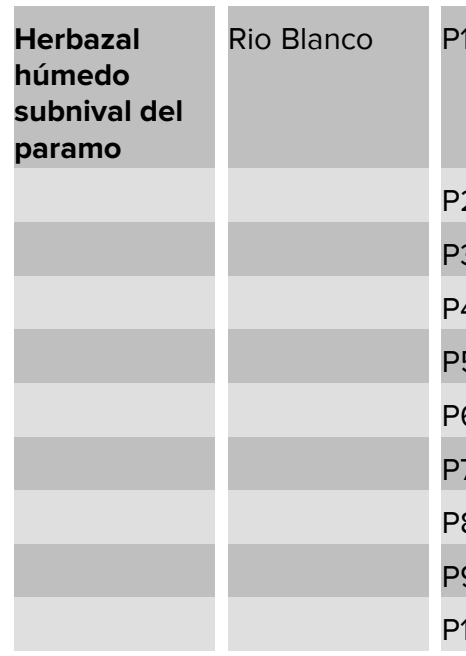

P1
P2
P3
P4
P5
P6
P7
P8
P9
P10

746251
746242
746380
746902
746847
746263
746333
746281
746307
746299

\begin{tabular}{l|l|}
\hline 9846963 & 4349 \\
\hline 9846954 & 4349 \\
\hline 9846936 & 4488 \\
9846966 & 4488 \\
\hline 9846921 & 4355 \\
\hline 9846844 & 4349 \\
\hline 9847329 & 4349 \\
\hline 9846916 & 4355 \\
\hline 9846909 & 4359 \\
\hline 9846854 & 4349 \\
\hline
\end{tabular}



Figure 1

Mapa de los sitios de muestreo en la comunidad Rio Blanco. 


\subsection{Diseño de muestreo}

La metodología utilizada fue la propuesta por [31] para el proyecto GLORIA en la región europea, a la que se le realizaron algunas variaciones que permitieron adaptarla a la zona de estudio [32, 33].

Se aplicaron los criterios: vegetación, composición vegetal y estado de conservación [34] para definir las zonas de muestreo además se utilizaron la ayuda de 128 ortofotos de la Reserva de Producción de Fauna Chimborazo. En los sitios de estudio se instalaron cuadrantes de $5 \times 5 \mathrm{~m}$ con un área de $25 \mathrm{~m} 2$ en total, cada cuadrante se subdividió en parcelas de 1×1 m, la recolección de información se realizó únicamente en los extremos o esquinas de cada parcela en los cuadrantes de $5 \times 5 \mathrm{~m}$ ya que los otros pueden quedar alterados por el pisoteo de los investigadores al realizar el muestreo. Cada una de las parcelas de 1×1 m, se subdividieron en cuadriculas de 0.1×0.1 m, para ello se utilizó un armazón de madera con un enrejado formado por hilos finos que delimitan 100 celdillas de 0,1×0,1 m, de acuerdo a la metodología del manual GLORIA y modificada por [33], con el fin de obtener la mayor cantidad de datos para el análisis.

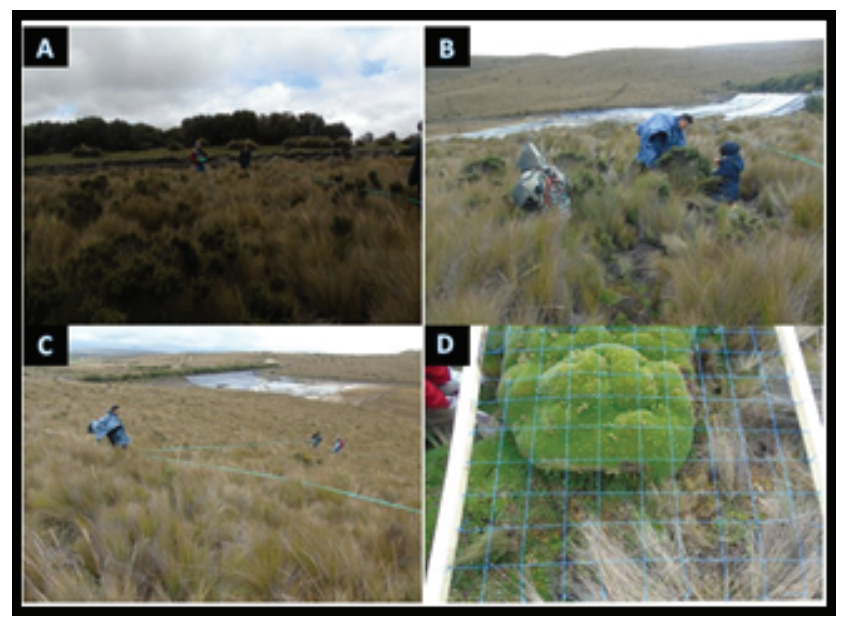

Figure 2

Fotografías ilustrativas de la formación vegetal en el área de estudio. (A) Zonificación de la formación vegetal, (B) Selección de los sitios de muestreo, (C) Delimitación de la parcela, (D) Inventariación de las especies.

En el interior de las parcelas se registró información relativa al número de especies y la cobertura de cada una de ellas, lo que permitió determinar la diversidad por familia, densidad e índices de diversidad. También se determinó el índice de valor de importancia (IVI), para lo cual se obtuvo previamente la dominancia y frecuencia relativa [35]. 


\subsection{Determinación de amenazas significativas que se encuentran en la formación vegetal}

El objeto de análisis fue el ecosistema herbazal húmedo subnival de páramo en la comunidad Río Blanco, para el cual se basó en la metodología de Planificación para la conservación de sitios [36], en el cual se detalla el análisis del objeto en función a la composición, función y extensión, las presiones se identificaron con base en severidad (nivel de daño al objeto de manejo) y alcance (superficie afectada), y finalmente las fuentes de presión a partir de dos criterios; contribución (aporte de la fuente actuando sola) e irreversibilidad (nivel de reversión de sus efectos).

\subsection{Lineamientos para la conservación de la formación vegetal}

Los lineamientos de conservación se formularon aplicando el método de investigación de campo, la técnica de diagramación de amenazas- estrategias, las orientaciones para desarrollar el análisis valorativo de amenazas de objetos de conservación propuesto por [37] y los lineamientos de estructuración de información formulados por [38].

\subsubsection{Análisis estadístico}

Los datos se analizaron acorde a las características para la estimación de la riqueza de especies, siendo la riqueza de especies, definida como el número de especies que se encuentran en un sitio determinado, o que se registran dentro de un muestreo, con el fin de determinar si el muestro es suficiente para incluir la mayoría de especies que existen efectivamente en un área. Por ello para evaluar la riqueza de especies se empleó la curva de acumulación de especies que muestra cómo el número de especies se va acumulando en función del número acumulado de muestras [39]. Hasta llegar a la asíntota, parar obtener la curva se ingresaron los datos en el software estimate.

\subsection{2. Índices de biodiversidad}

Se configuro un listado de especies con sus respectivas coberturas (400\%) en cada una de las parcelas, posteriormente se obtuvo: riqueza, diversidad (índice de Simpson, índice de Shannon) para ello se utilizó el software estadístico estimate

Según [35], la fórmula para el índice de Simpson es:

$$
D_{s i}^{\prime}=\sum_{i=1}^{S} \frac{n_{i}-1}{n-1}
$$

donde: 
n: el número total de organismos de una especie en particular; $\mathrm{N}$ : el número total de organismos de todas las especies.

El valor de $D$ oscila entre 0 y 1 :

Si el valor de $\mathrm{D}$ da 0 , significa diversidad infinita.

Si el valor de D da 1, significa que no hay diversidad.

El índice es una representación de la probabilidad de que dos individuos, dentro de una misma región y seleccionados al azar, sean de la misma especie. El rango del índice de Simpson va de 0 a 1, así:

Cuanto más se acerca el valor de D a 1, menor es la diversidad del hábitat. Cuanto más se acerca el valor de $\mathrm{D}$ a 0 , mayor es la diversidad del hábitat.

Es decir, cuanto mayor es el valor de D, menor es la diversidad. Esto no es fácil de interpretar de manera intuitiva y podría generar confusión, razón por la cual se llegó al consenso de restar el valor de D a 1, quedando de la siguiente manera: 1-D.

\subsubsection{Indice De Shannon - Wiever}

Es el índice más usado, expresa la uniformidad de los valores de importancia a través de todas las especies de la muestra. Mide el grado promedio de incertidumbre en predecir a que especie pertenecerá un individuo escogido al azar de una colección. Asume que los individuos son seleccionados al azar y que todas las especies de una comunidad están representadas en la muestra.

Adquiere valores entre cero cuando hay una sola especie y el logaritmo de S cuando todas las especies están representadas por el mismo número de individuos. Se puede calcular usando el logaritmo natural (más exacto) o con logaritmo base 10

El índice de Shannon y Weaver (1949 se basa en la teoría de la información (mide el contenido de información por símbolo de un mensaje compuesto por ' $S$ ' clases de símbolos discretos cuyas probabilidades de ocurrencia son pi...pS) y es probablemente el más usado en ecología de comunidades.

Integra dos componentes: [39]

Riqueza de especies - Equitatividad/representatividad (dentro del muestreo).

La fórmula para su cálculo es:

$$
H=\sum_{i=1}^{S}(P i)\left(\log _{n} P i\right),
$$

donde:

HI: Índice de Shannon; Ln: Logaritmo natural;

Pi: Proporción del número total de individuos que constituye la especie i. 


\section{Resultados y Discusión}

\subsection{Esfuerzo de muestreo}

Se utilizó la curva de acumulación de especies para validar el esfuerzo de muestreo [40], de acuerdo con los datos tabulados mediante el software Eco Sim teniendo en cuenta la abundancia, riqueza, límite inferior y límite superior con un porcentaje de confiabilidad del 95\%, la tendencia de la curva de acumulación de especies alcanza la asíntota con un valor de 1400 individuos, por lo que se considera que el muestreo fue suficiente para representar la composición florística lo que concuerda con los dicho por [41] cuanto mayor sea este esfuerzo, mayor será el número de especies colectadas.

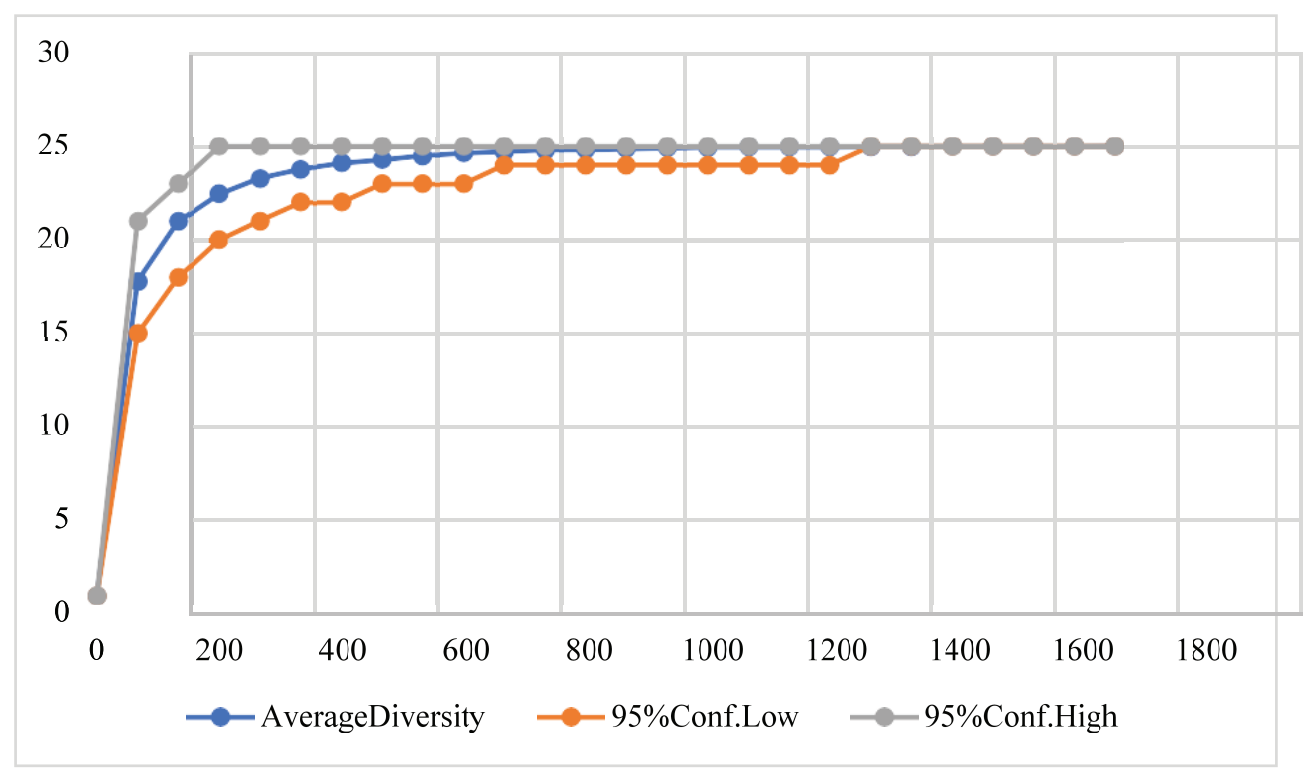

Figure 3

Curva de acumulación de especies para validar el esfuerzo de muestro en la formación vegetal.

\subsection{Riqueza florística y cobertura}

Se registró un total de 25 especies, 10 familias, 23 géneros y 10 órdenes en el área de estudio, las que mayor número de especies tienen son: Asteraceae (10) y Geraniaceae (4), el resto de familias con valores menores. Estas dos familias contienen 14 especies es decir el $56 \%$ del total de familias registradas.

Calamagrostis intermedia, Plantago rigida, Geranium ayavacense tienen presencia en las 10 zonas de muestreo, Calamagrostis intermedia tiene mayor cobertura. Hypochaeris sonchoides tiene presencia en nueve de las 10 zonas de muestreo, las especies restantes registran presencias y coberturas en menor representatividad. Lo 
que concuerda con estudios realizados por [18, 22] que indican que son las especies más características en zonas de paramo debido a la resiliencia que poseen para resistir en zonas y condiciones adversas.

Table 2

Listado de especies más representativas con sus respectivas coberturas (400\%) en las 10 parcelas.

\begin{tabular}{|c|c|c|c|c|c|c|c|c|c|c|}
\hline Plantago rigida Kunth & 52 & 2 & 146 & 150 & 28 & 60 & 51 & 56 & 20 & 83 \\
\hline $\begin{array}{l}\text { Geranium ayavacense W. } \\
\text { (Pasuchaca) }\end{array}$ & 49 & 51 & 71 & 13 & 20 & 10 & 18 & 16 & 36 & 3 \\
\hline Hypochaeris sessiliflora Kunth & 5 & 0 & 0 & 0 & 0 & 8 & 1 & 0 & 0 & 2 \\
\hline $\begin{array}{l}\text { Lachemilla hispidula (L. M. Perry) } \\
\text { Rothm }\end{array}$ & 200 & 4 & 0 & 0 & 0 & 13 & 6 & 44 & 7 & 15 \\
\hline $\begin{array}{l}\text { Disterigma empetrifolium (Kunth) } \\
\text { Drude }\end{array}$ & 1 & 0 & 0 & 5 & 58 & 8 & 0 & 4 & 0 & 3 \\
\hline Hypochaeris sonchoides Kunth & 2 & 1 & 68 & 86 & 38 & 12 & 0 & 12 & 1 & 1 \\
\hline $\begin{array}{l}\text { Xenophyllum humile (Kunth) } \\
\text { V.A.Funk }\end{array}$ & 25 & 5 & 0 & 0 & 4 & 0 & 20 & 0 & 0 & 30 \\
\hline $\begin{array}{l}\text { Monticalia andicola (Turcz.) C. } \\
\text { Jeffrey }\end{array}$ & 5 & 0 & 0 & 0 & 0 & 0 & 0 & 0 & 0 & 0 \\
\hline $\begin{array}{l}\text { Azorella pedunculata (Spreng.) } \\
\text { Mathias \& Constance }\end{array}$ & 0 & 90 & 0 & 0 & 0 & 30 & 0 & 10 & 0 & 56 \\
\hline Gentianella cernua (Kunth) & 0 & 2 & 0 & 1 & 0 & 8 & 22 & 9 & 7 & 0 \\
\hline Niphogeton dissecta (Benth.) & 0 & 4 & 8 & 10 & 1 & 22 & 0 & 7 & 9 & 0 \\
\hline $\begin{array}{l}\text { Pentacalia peruviana (Pers.) C. } \\
\text { Jeffrey }\end{array}$ & 0 & 1 & 0 & 0 & 0 & 19 & 46 & 0 & 37 & 0 \\
\hline $\begin{array}{l}\text { Huperzia crassa (Humb. \& Bonpl. } \\
\text { ex Willd.) Rothm. }\end{array}$ & 0 & 5 & 5 & 0 & 0 & 10 & 0 & 0 & 0 & 0 \\
\hline Valeriana microphylla Kunth & 0 & 1 & 0 & 0 & 0 & 5 & 0 & 0 & 47 & 0 \\
\hline Loricaria thuyoides (Lam.) Sch Bip & 0 & 0 & 0 & 55 & 17 & 0 & 0 & 0 & 0 & 0 \\
\hline Halenia weddelliana Gilg. & 0 & 0 & 0 & 10 & 0 & 1 & 0 & 1 & 0 & 0 \\
\hline Baccharis caespitosa (Lam.) Pers. & 0 & 0 & 0 & 12 & 20 & 5 & 30 & 24 & 58 & 10 \\
\hline Geranium multipartitum Kunth & 0 & 0 & 0 & 10 & 0 & 11 & 32 & 5 & 3 & 0 \\
\hline Lupinus microphyllus Desr. & 0 & 0 & 0 & 7 & 20 & 0 & 28 & 19 & 10 & 6 \\
\hline Gentiana sedifolia Kunth & 0 & 0 & 0 & 0 & 0 & 7 & 0 & 0 & 0 & 0 \\
\hline Lucilia kunthiana (DC.) Zardini & 0 & 0 & 0 & 0 & 0 & 6 & 0 & 0 & 0 & 11 \\
\hline $\begin{array}{l}\text { Lachemilla orbiculata (Ruiz \& Pav.) } \\
\text { Rydb }\end{array}$ & 0 & 0 & 0 & 0 & 0 & 7 & 34 & 28 & 24 & 14 \\
\hline Chuquiraga jussieui J.F.Gmel. & 0 & 0 & 0 & 0 & 0 & 0 & 30 & 38 & 26 & 49 \\
\hline ESPECIES & P1 & P2 & P3 & P4 & P 5 & P6 & P7 & P8 & P9 & P10 \\
\hline $\begin{array}{l}\text { Calamagrostis intermedia(J. Presl) } \\
\text { Steud }\end{array}$ & 232 & 15 & 90 & 31 & 89 & 143 & 82 & 122 & 115 & 70 \\
\hline $\begin{array}{l}\text { Oritrophium peruvianum (Lam.) } \\
\text { Cuatrec. }\end{array}$ & 0 & 0 & 0 & 0 & 0 & 0 & 0 & 0 & 0 & 20 \\
\hline
\end{tabular}




\section{3. Índices de biodiversidad}

La biodiversidad de la Reserva es la variabilidad que existe entre los organismos, desde las fuentes y sistemas ecológicos de los cuales forman parte, incluyendo la diversidad dentro de las especies, entre especies y la de las formaciones vegetales como ecosistemas.

Desde la perspectiva de la Ecología, las unidades de estudio son paisajes, ecosistemas, comunidades y conjuntos [42,43], reconociéndose que no todas las especies son abundantes.

En muchos ecosistemas, algunas especies monopolizan el espacio o la energía (tienen la mayor parte de la biomasa o de los individuos), en tanto que muchas otras tienen muy pocos individuos o biomasa [44].

\subsection{1. Índice de Simpson}

El listado de especies se ingresó en el software estadístico PAST generándose el índice de diversidad de Simpson que arrojo valores de 0,92 lo cual indica que el la biodiversidad es alta de acuerdo a [35], este resultado difiere de estudios realizados anteriormente en la zona los cuales evidencian que la diversidad florística va de media a baja, lo cual coinciden con algunos estudios realizados en la zona como el de [34].

\subsection{2. Índice de Shannon-Weaver}

Aguirre Mendoza [39] asume que los individuos son seleccionados al azar y que todas las especies de una comunidad están representadas en la muestra, se configuro un listado de especies con sus respectivas coberturas en cada una de las parcelas, posteriormente fueron calculados en el software estadístico past obteniéndose $(2,82)$, de acuerdo al índice de diversidad especifica de Shannon-Weaver su valor normal está entre 2 y 3 donde valores inferiores a 2 se consideran bajos en diversidad y superiores a 3 son altos en diversidad de especies, se infiere que existe una diversidad media en la zona de estudio, tal como lo planteo [34] en su investigación.

Ante las demás especies, no obstante, según menciona que la biodiversidad disminuye con la altitud y la latitud así este ecosistema no deja de ser importante no solo porque alberga a una gran cantidad de avifauna sino por todos los servicios ecosistémicos que brinda. 


\subsection{3. Índice de valor de importancia}

Mediante el índice de valor de importancia se obtuvieron las especies con el mayor peso ecológico, siendo cinco las especies más representativas pues abarcan el 46,93\% del IVI total, estas especies; G. ayavacense (12,44\%), P. rigida (9,48\%), Calamagrostis intermedia $(9,28 \%)$, Lachemilla hispidula $(8,87 \%)$ y $H$. sonchoides $(6,86 \%)$, desempeñan un papel importante en la tipología de la vegetación [45].

\subsection{Amenazas significativas}

Se definió como objeto de análisis a la formación vegetal herbazal húmedo subnival de páramo de la comunidad Rio Blanco de (a) categoría natural; (b) características físicas el objeto de análisis se encuentra a una altitud de 4330 a 4350 m.s.n.m., posee una temperatura de $5^{\circ} \mathrm{C}$; (c) valoración, la sensibilidad al cambio en este ecosistemas es muy alta y se encuentra en proceso de deterioro; presentan una importancia intrínseca por su conectividad (Prácticas productivas ancestrales, Páramo herbáceo) y por su representatividad (Páramo seco, Microcuenca).

En cuanto a servicios ecosistémicos, la formación vegetal brinda servicios de suministro, regulación y cultural. Además, la presencia de fauna es media y la de flora nativa alta. Adicionalmente el uso actual del objeto de conservación es económico.

\subsubsection{Presiones y fuentes de presión a la formación vegetal herbazal húmedo subnival del paramo}

Las actividades generadas por los habitantes como las quemas de pajonal, sobrepastoreo, erosión y compactación del suelo por ganadería de suelo han ocasionado la destrucción y deterioro del hábitat (ponderación de 2,8

= alta), impulsado por actividades de subsistencia incompatible con el ecosistema como la presencia de ganado bovino y ovino, actividades antrópicas generadas por el hombre, desconocimiento al manejo adecuado del páramo y la presencia de desechos inorgánicos (ponderación de 2,05 = media), lo que concuerda con estudios realizados antes en la zona de amortiguamiento de la Reserva de Producción de Fauna Chimborazo como el de [9].

\subsection{Lineamientos para la conservación de la formación vegetal}

Para moderar las presiones y disminuir las fuentes de presión se generaron participativamente estrategias con el fin de Mantener la estructura, la composición y la funcionalidad del ecosistema herbazal húmedo subnival de páramo de la comunidad de 
Table 3

Densidad, frecuencia e índice de valor de importancia de las especies registradas.

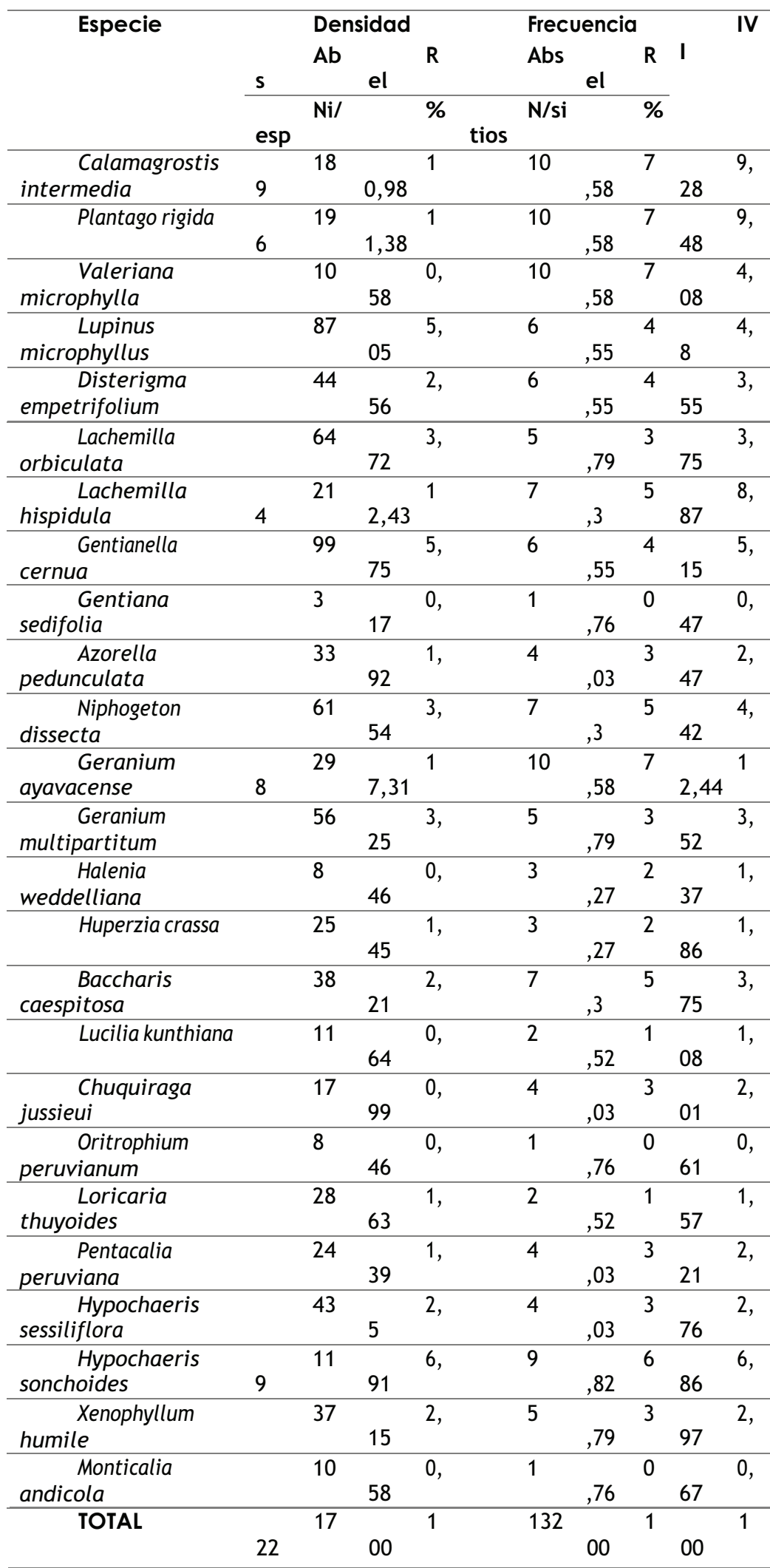

Abs: Valores absolutos; Rel: Valores relativos (\%). 
Table 4

Calificación presiones.

Presiones
Erosión del Suelo
Sobrepastoreo
Quema del pajonal
Compactación del suelo por
ganadería Presión global

Severidad
Muy alto $(4)$
Alto $(3,5)$
Alto $(3,5)$
Medio $(2,5)$

\begin{tabular}{l|l} 
Alcance & Presión \\
\hline Alto $(3,5)$ & Muy Alto $(3,8)$ \\
Medio $(2,5)$ & Alto $(3)$ \\
\hline Medio $(2,5)$ & Alto $(3)$ \\
Bajo $(2,2)$ & Medio $(2,4)$ \\
& Alto $(2,8)$
\end{tabular}

Table 5

Calificación fuentes de presión.

\begin{tabular}{|c|c|c|c|}
\hline Fuente de presión & Contribución & Irreversibilidad & Fuente de presión \\
\hline $\begin{array}{l}\text { Presencia de ganado } \\
\text { bovino y ovino }\end{array}$ & Alto (3) & Medio (2) & Medio $(2,5)$ \\
\hline $\begin{array}{l}\text { Actividades antrópicas } \\
\text { generadas por el } \\
\text { hombre }\end{array}$ & Alto $(3,5)$ & Medio $(2,5)$ & Alto (3) \\
\hline $\begin{array}{l}\text { Desconocimiento al } \\
\text { manejo adecuado del } \\
\text { páramo }\end{array}$ & Alto (3) & Medio $(2,5)$ & Alto $(2,8)$ \\
\hline $\begin{array}{l}\text { Presencia de } \\
\text { desechos inorgánicos }\end{array}$ & Medio (2) & Medio (2) & Medio (2) \\
\hline $\begin{array}{l}\text { Total de las fuentes } \\
\text { de presión }\end{array}$ & & & Medio $(2,05)$ \\
\hline
\end{tabular}

Rio Blanco del que se derivan la ejecución de estrategias como Programa de Control y Vigilancia; que permitirá la Programas Comunicación, Educación y Participación Ambiental, Programa de buenas prácticas agropecuarias, que se aplicarían durante lograrían en un periodo comprendido de 3 a 5 años la disminución de sobrepastoreo, disminución de la compactación del suelo por ganadería, disminución de las quemas de pajonal y la disminución de la erosión del suelo.

\section{Conclusiones}

La formación vegetal herbazal húmedo subnival de páramo está compuesta por herbazales dispersos que se encuentran restringidos a las partes más altas de las montañas, se evidencia la dominancia de Calamagrostis intermedia, P. rigida, G. ayavacense en las 10 zonas de muestreo. Que son los grupos botánicos caracterizaos por la elevada resistencia a las condiciones climáticas adversas y a los procesos de degradación del páramo en la Reserva de Produccion de Fauna Chimborazo.

Se infiere que los valores medios y altos que se obtuvieron al aplicar los índices de biodiversidad Simpson 0,92 y shannon-Weaver 2,83 , se deben al elevado número de 


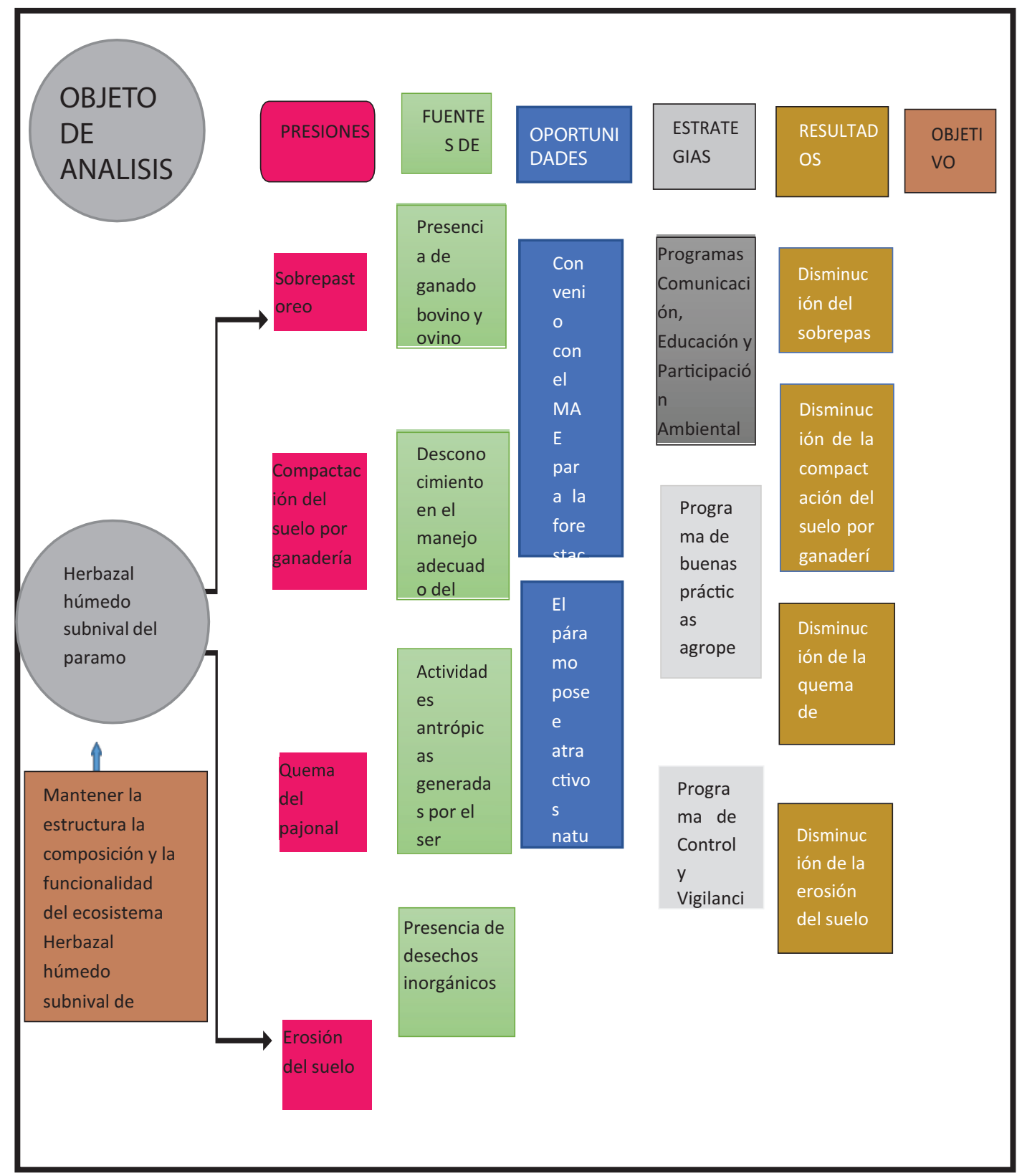

Figure 4

Objetivos y estrategias del objeto de análisis.

especies que se encontraron en la mayoría de parcelas pertenecientes a la especie Calamagrostis intermedia. Se muestrearon 1400 especies que fueron suficientes para validar el esfuerzo de muestreo.

La distribución de las especies en el área de estudio presenta una fisonomía típica de ellas evolucionando en formas de roseta basal, reduciendo su tamaño, pero aumentando el número de individuos por especie, presentando en casos simbiosis entre ellas como una estrategia de adaptación al cambio climático la cual no ha escapado este sitio de estudio. 
Las presiones a la formación vegetal herbazal húmedo subnival arrojaron un puntaje de 2,8, que corresponde a la categoria: Alto, refleja la afectacion del objeto focal de conservacion en muchos sectores de la formacion vegetal alcanzando un puntaje correspondiente a severidad y alcance $(2,8)$ que se categorizan como alto, afectando principalmente a los servicios ecosistemicos de regulación hídrica y suministro de agua.

Para mitigar las amenazas que intervienen en la formación vegetal herbazal húmedo subnival de páramo en el área de estudio, se han generado de manera participativa lineamientos a ser trabajadas en conjunto con los gestores del area protegida como: Programa de Control y Vigilancia, Programa de Comunicación, Educación y Participación Ambiental, Programa de buenas prácticas agropecuarias, las cuales ayudarían a disminuir la degradación del objeto de estudio.

Debido a la gran presencia de la especie Calamagrostis intermedia típica del área de estudio esta formación se convierte en una de las más importantes para realizar un monitoreo ante el creciente cambio climático ya que el páramo es un gran sumidero de carbono, no tanto por la biomasa que sustenta, como por las formaciones edáficas que alberga. En efecto, el pajonal del páramo puede cuantificarse en unas 40 tn/ha, como máximo, de materia seca, lo que equivale a unas 20 tn/ha de carbono.

\section{Agradecimientos}

Al Rector de la ESOCH Dr, Byron Vaca Barahona por el apoyo institucuonal para la ejecucion del proyecto. Al Ing. Eduardo Muoz Director del proyecto 'Medidas ante los riesgos que afrontan los ecosistemas de la Reserva de Produccion de Fauna Chimborazo frente al cambio de uso de suelo' de la Escuela Superior Politécnica del Chimborazo por haber confiado en el equipo para la ejecucion del proyecto.

\section{References}

[1] Jones K, Venter O, Fuller R et al. One-third of global protected land is under intense human pressure. Science. 2018;360: 788-791.

[2] Palomo I, Montes C, Martín-López B et al. Incorporating the social-ecological approach in protected areas in the anthropocene. BioScience. 2014;64: 181-191.

[3] Pérez M, Flores J. Uso público de áreas protegidas. Una alternativa para la participación comunitaria. Scielo. 2016;8:138-145.

[4] Salgado S, Cuesta F, Beltrán K, León S, Romoleroux K, Ortiz E, Cárdenas A, Velástegui A. Distribución espacial, sistemas ecológicos y caracterización climática en el Ecuador, EcoCiencia, Proy. Páramo Andin. y Herb. Quito: QCA; 2009. 
[5] Oldekop J, Holmes G, Harris W, Evans K. A Global assessment of the social and conservation outcomes of protected areas. Conserv Biol. 2016;30(Suppl1):133-141.

[6] Riemann H, Santes-Álvarez R, Pombo A. The role of natural protected areas in local development: The case of the peninsulaof baja California. Gest. y Polit. Publica. 2011;20:141-172.

[7] Qin S, Golden R, Cook C et al. Protected area downgrading, downsizing, and degazettement as a threat to iconic protected areas. Conserv. Biol. 2019;33:12751285.

[8] Szymanski M. Portal Unión Internacional para el Cuidado de la Naturaleza; 2016. Disponible en: https://www.iucn.org/es/news/secretariat/201609/el-15-de-las-tierr as-del-planeta-están-protegidas-pero-quedan-excluidas-áreas-cruciales-para-la-b iodiversidad

[9] Lozano P, Armas A, Machado V. Estrategias para la conservación del ecosistema páramo en Pulinguí San Pablo y Chorrera Mirador, Ecuador. Enfoque UTE. 2016;7: 55-70.

[10] Muñoz B. Percepciones de la gestión del turismo en dos reservas de biosfera ecuatorianas: Galápagos y Sumaco. Investig. Geogr. 2017;110-125.

[11] Morales-Betancourt J, Estévez-Varón J. El páramo: ¿ecosistema en vía de extinción? Revista Luna AzuL. 2006;22:39-51.

[12] Hofstede R, Vasconez S, Cerra M. Vivir en los páramos. Percepciones, vulnerabilidades, capacidades y gobernanza ante el cambio climático. Quito: UICN; 2015.

[13] Rodríguez N, de la Nuez AM. La vicuña ecuatoriana y su entorno Ecuador mamallaktapi tiyak wikuña paypak kuskapi. $1^{\text {st }}$ edition. Riobamba; 2017.

[14] Lozano P. Valoración económica del carbono capturado en el suelo de los bofedales de la Reserva de Producción de Fauna Chimborazo [tesis de posgrado]. Riobamba: ESPOCH; 2017.

[15] Sistema de clasificación de los Ecosistemas del Ecuador Continental 2013 (Ecuador).

[16] Hofstede R, Calles J, López V et al. Los páramos andinos ¿Qué Sabemos?. Quito: UICN; 2014

[17] Actualizacion del Plan de Manejo de la Reserva de Produccion de Fauna Chimborazo 2014 (Ecuador).

[18] Bustamante M, Albán M, Argüello M. Los Paramos de Chimborazo Un Estudio Socioambiental para la toma de Decisiones. Quito: EcoCiencia; 2011.

[19] Josse C, Navarro G, Comer P et al. Sistemas ecológicos de América Latina y El Caribe: Una clasificación de trabajo de los sistemas terrestres., Arlington: NatureServe; 2003.

[20] Körner C. Alpine Plant Life: Functional ecology of high mountain ecosystem plants. The climate plants experience. Heidelberg: Springer; 2003. p. 31-46. 
[21] Sklenář P, Balslev H. Superpáramo plant species diversity and phytogeography in Ecuador. Flora - Morphol. Distrib. Funct. Ecol. Plants. 2005;200:416- 433.

[22] Sklenář P. Presence of cushion plants increases community diversity in the high equatorial Andes. Flora - Morphol. Distrib. Funct. Ecol. Plants. 2009;204: 270-277.

[23] Molina R, Campos R, Guerrero HS, Giraldo L, Atzori A. Sustainable feedbacks of colombian paramos involving livestock, agricultural activities, and sustainable development goals of the agenda 2030. Systems. 2019;7:52-69.

[24] Farley K, Bremer L. Water is life: Local perceptions of páramo grasslands and land management strategies associated with payment for ecosystem services. Ann. Am. Assoc. Geogr. 2017;107:1-11.

[25] Estadísticas de Patrimonio Ecuador continental 2015(Ecuador).

[26] Giné D, Sánchez R. El Páramo Andino: Características territoriales y estado ambiental. aportes interdisciplinarios para su conocimiento1. Estudios Geograficos. 2015;76:369-393.

[27] Hofstede R. Health state of Páramos: An effort to correlate science and practice. Lyonia. 2004;6:61-73.

[28] Pauli H, Gottfried M, Lamprecht A et al. Manual para el trabajo de campo del proyecto GLORIA. Aproximación al estudio de las cimas. Métodos básico, complementarios y adicionales. $5^{\text {th }}$ ed. Jaca: GLORIA; 2015.

[29] The Nature Conservancy. 2016.

[30] Ministerio del Ambiente del Ecuador. Plan Gerencial Reserva de Producción Faunistica Chimborazo Resumen Ejecutivo. Riobamba; 2002.

[31] Pauli H, Gottfried M, Hohenwallner D, Reiter K, Grabherr G. Manual para el trabajo de campo del proyecto GLORIA. Aproximación al estudio de las cimas. Iniciativa para la Investigación y el Seguimiento Global de los Ambientes Alpinos, como contribución al Sistema Terrestre de Observación Global (GTOS). Jaca: 2003.

[32] Eguiguren V, Ojeda T, Aguirre N. Diversidad Florística del ecosistema paramo del Parque Nacional Podocarpus para el monitoreo del Cambio Climático. Ecologia Forestal. 2010;1:7-18.

[33] Rodriguez, M. Estudio de la diversidad florística a diferentes altitudes en el páramo de almohadillas de la comunidad yatzaputzán, cantón Ambato. 2011.

[34] Caranqui J, Lozano P, Reyes J. Composición y diversidad florística de los páramos en la Reserva de Producción de Fauna Chimborazo, Ecuador. Enfoque UTE. 2016;7:3345.

[35] Aguirre M. Guia de metodos para medir la biodiversidad. Loja: 2013.

[36] The Nature Conservancy, 2006.

[37] Granizo, et al, 2006. 
[38] Ortegón, E, Pacheco J, Prieto A. Metodología del marco lógico para la planificación, el seguimiento y la evaluación de programas y proyectos. Santiago de Chile: CEPAL; 2015

[39] Pauta L. Biodiversidad fungica en el suelo del bosque protector Aguarongo. Azuay. 2016.

[40] González-Oreja J, De la Fuente-Díaz-Ordaz A, Hernández-Santín L, Buzo-Franco $D$, Bonache-Regidor C. Evaluación de estimadores no paramétricos de la riqueza de especies. Un ejemplo con aves en áreas verdes de la ciudad de Puebla, México. Anim. Biodivers. Conserv. 2010;33: 31-45.

[41] Jiménez-Valverde A, HJ. Las curvas de acumulación de especies y la necesidad de evaluar la calidad de los inventarios biológicos. Rev. Ibérica de Aracnología. 2003;8:151-161.

[42] Wilson 1991.

[43] Matthews y Whittaker 2014.

[44] McGill, 2011.

[45] Soler P, Berroterán J, Gil J, Acosta R. Índice valor de importancia, diversidad y similaridad florística de especies leñosas en tres ecosistemas de los llanos centrales de Venezuela. Agron. Trop. 2011;62:25-37.

[46] Geoinformática y Sistemas Cia. Ltda. "Estudio del estado actual del ecosistema páramo en tungurahua", honorable gobierno provincial de tungurahua (HGPT) Deutsche Gesellschaft für Internationale Zusammenarbeit (GIZ) GmbH (Cooperación Alemana para el Desarrollo). Ambato: 2015. 